\title{
François-Charles MOUGEL, Les Élites britanniques de la Glorieuse Révolution à Tony Blair
}

Paris : Ellipses, 2005

Jacques Carré

\section{OpenEdition}

\section{Journals}

Édition électronique

URL : http://journals.openedition.org/rfcb/1650

DOI : $10.4000 /$ rfcb. 1650

ISSN : 2429-4373

Éditeur

CRECIB - Centre de recherche et d'études en civilisation britannique

Édition imprimée

Date de publication : 1 janvier 2006

ISSN : 0248-9015

\section{Référence électronique}

Jacques Carré, «François-Charles MOUGEL, Les Élites britanniques de la Glorieuse Révolution à Tony

Blair », Revue Française de Civilisation Britannique [En ligne], XIII-4 | 2006, mis en ligne le 01 janvier 2006, consulté le 25 septembre 2020. URL : http://journals.openedition.org/rfcb/1650 ; DOI : https://doi.org/ $10.4000 /$ rfcb. 1650

Ce document a été généré automatiquement le 25 septembre 2020

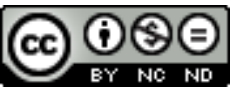

Revue française de civilisation britannique est mis à disposition selon les termes de la licence Creative Commons Attribution - Pas d'Utilisation Commerciale - Pas de Modification 4.0 International. 


\section{François-Charles MOUGEL, Les Élites britanniques de la Glorieuse Révolution à Tony Blair}

Paris : Ellipses, 2005

Jacques Carré

\section{RÉFÉRENCE}

François-Charles MOUGEL, Les Élites britanniques de la Glorieuse Révolution à Tony Blair.

Paris : Ellipses, 2005, 157p. ISBN 2729823158.

1 Ce petit ouvrage de la collection "Les essentiels de la civilisation britannique " présente en neuf chapitres denses et alertement rédigés beaucoup plus qu'un panorama des élites au pouvoir sur un peu plus de trois siècles. C'est une réflexion stimulante sur ce qui a fait du Royaume-Uni, selon les termes de l'auteur, « un modèle d'évolution politique et sociale, au long d'un processus exceptionnel de continuité et de stabilité » (p.9). F.-C. Mougel évoque brièvement dans l'introduction les différentes théories des élites (sans mentionner, et c'est dommage, les textes canoniques sur le sujet, comme ceux de Wright Mills) et laisse au lecteur le soin de discerner peu à peu celle qui lui paraît la plus pertinente dans le cas britannique. La première partie, portant sur le long XVIII ${ }^{e}$ siècle, insiste sur l'événement fondateur qu'a été la Révolution de 1688, judicieusement décrite en termes de "pacte " à la fois politique, économique et social, aboutissant à une stabilisation des rapports entre Couronne, élites terriennes, intérêts commerciaux et un peuple encore politiquement "virtuel ». Les ressorts du pouvoir de l'oligarchie whig au XVIII ${ }^{\mathrm{e}}$ siècle sont décrits avec beaucoup de précision, et sa capacité à intégrer certains membres de la bourgeoisie d'affaires dès cette époque est soulignée. La médiation de la culture et de la civilité dans cette intégration est justement notée. On regrettera seulement que pour cette période le rôle de l'Église établie dans le fonctionnement du système de pouvoir élitaire ne soit pas 
suffisamment pris en compte (même si on ne partage pas forcément le point de vue extrême de J.C.D. Clark sur la question).

2 La deuxième partie, portant sur l'époque victorienne et édouardienne, est centrée sur l'idée de l'adaptabilité du groupe élitaire ancien, qui prend en compte l'apparition d'élites industrielles, et s'implique dans ce que l'auteur appelle 'gentlemanly capitalism'. F.-C. Mougel fournit une riche information sur la nouvelle donne économique et le processus d'évolution des institutions. Il choisit de présenter l'extension progressive du droit de vote comme une stratégie habile et délibérée des élites dirigeantes : «Parce qu'il est capable de le structurer dans des termes acceptables par lui, le système élitaire ne sera pas la victime du changement» (p.61). Cette interprétation n'est assurément pas la seule possible. D'aucuns ont avancé que cette extension du droit de vote résultait en réalité de concessions nullement choisies, mais imposées par des pressions diverses, comme, dans les années 1830 , celles des différents groupes radicaux soutenus par une partie de l'opinion. Mais l'auteur ne parle presque jamais (sauf dans la conclusion) de concessions des élites, et préfère le terme "accommodement", dès qu'il s'agit d'aborder un conflit potentiel entre elles et les autres éléments du corps politique et social. Il insiste sur la capacité des élites britanniques à canaliser les affrontements possibles avec «l'offre idéologique ", voire avec l'opinion ouvrière, vers la structure parlementaire. Idée intéressante, qui contribuerait à expliquer la quasi-sacralisation victorienne du Parlement. L'auteur propose même de voir dans le «modèle de Westminster » triomphant du début du XXe siècle ce qui a finalement empêché le parti travailliste naissant de devenir une véritable contre-élite, et a fait de lui une "élite associée ».

3 La troisième partie, portant sur la période 1914-2005, évoque la diversification croissante des élites, avec la montée en puissance des "élites de socialisation" (patrons de presse notamment), des « élites d'État " (la haute administration), et d'une "classe d'affaires" de plus en plus puissante, qui contribuèrent à la naissance du 'Corporate State'. Dans une perspective sociologique, l'auteur discerne dans la période de l'entre-deux-guerres une "médiation élitiste qui canalise vers le haut la mobilité sociale au bénéfice de l'upper class» (p.106). Cette analyse est développée plus loin, chiffres à l'appui, à propos de l'après-guerre, pour illustrer le "profond décalage existant entre la stratification sociale globale et l'ancrage sociologique des élites » (p. 124). Mais curieusement, le concept de « méritocratie » n'est pas analysé. Les pages sur la période 1979-2005 (chapitre 9) sont les plus originales et les plus stimulantes de ce livre. Non sans quelque goût pour la provocation (mais pourquoi pas ?), F.-C. Mougel traite comme un tout cohérent l'action de Mrs Thatcher, John Major et Tony Blair. Il voit dans cette période, qui a vu le démantèlement du Corporate State et la présidentialisation du régime, la naissance d'un nouvel establishment reposant sur "Downing Street, la City et les Docklands, nouvelle base des médias nationaux " (p. 144). Sociologiquement, c'est la moyenne bourgeoisie des professions qui en fournit une bonne partie des acteurs, tandis que l'influence de l'old boy network recule sensiblement. C'est assurément un tournant majeur de la sociologie du pouvoir. Mais n'a-t-il pas commencé bien plus tôt ?

4 La conclusion ajoute à ces analyses fort bien documentées une interprétation de l'évolution des élites en termes de cycles: il y aurait dans chacune des périodes étudiées «trois phases successives de réorganisation, d'épanouissement et de transition» (p.154). Les lecteurs étudiants de cet ouvrage apprécieront sûrement la 
clarté de cette vaste fresque présentant les élites dans leurs mutations au cours de trois siècles. Les chercheurs aussi, même s'ils regretteront qu'un ouvrage aussi stimulant soit parfois elliptique et un peu avare de références bibliographiques.

\section{AUTEURS}

JACQUES CARRÉ

Université Paris IV-Sorbonne 